\title{
Presence of pseudopolyps in ulcerative colitis is associated with a higher risk for treatment escalation
}

\author{
Dimitrios S. Politis ${ }^{a}$, Konstantinos Papamichael ${ }^{b}$, Konstantinos H. Katsanos ${ }^{a}$, loannis Koulouridisc, \\ Despoina Mavromati ${ }^{\mathrm{d}}$, Epameinondas V. Tsianos ${ }^{\mathrm{a}}$, Dimitrios K. Christodoulou ${ }^{\mathrm{a}}$ \\ University of Ioannina, Greece; Beth-Israel Deaconess Medical Center, Harvard Medical School, Boston, \\ Massachusetts, USA; Tufts Clinical and Translational Science Institute, Tufts University, Boston, MA, USA; Berkshire \\ Medical Center, Pittsfield, MA, USA
}

\section{Abstract}

Background Pseudopolyps in ulcerative colitis (UC) are considered as indicators of previous episodes of severe inflammation and ulceration of the mucosa. The aim of the study was to investigate the long-term outcomes of patients treated for UC, with or without pseudopolyps.

Methods This was a retrospective single-center study. Consecutive patients with UC and available endoscopic data from 2000 until 2016 were eligible for the study and were followed until June 2017. Patients with incomplete medical/endoscopic charts or interrupted follow up were excluded from the study. Primary outcomes included time to treatment escalation, treatment escalation to biological agents or surgery, and UC-related hospitalization.

Results Eighty-three UC patients were included in the study, of whom $25(30 \%)$ had pseudopolyps. The median duration of follow up was 2.8 years (interquartile range: 1.1-4.9). Multiple Cox regression analysis identified the presence of pseudopolyps as the only variable independently associated with treatment escalation (hazard ratio [HR] 2.3, 95\% confidence interval [CI] 1.2-4.3; $\mathrm{P}=0.014$ ) and escalation to biological agents or surgery (HR 6.3, 95\%CI $1.9-20.7 ; \mathrm{P}=0.002)$.

Conclusion This retrospective single-center study provides the first preliminary evidence that patients with UC and pseudopolyps may represent a subpopulation with a higher inflammatory burden and a greater need for treatment escalation, including to biological agents or surgery. Large, prospective multicenter studies are certainly warranted to confirm these findings.

Keywords Pseudopolyps, ulcerative colitis, immunosuppression, biological treatment

Ann Gastroenterol 2019; 32 (2): 1-6

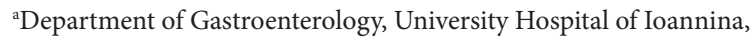
Faculty of Medicine, School of Health Sciences, University of Ioannina, Ioannina, Greece (Dimitrios S. Politis, Konstantinos H. Katsanos, Epameinondas V. Tsianos, Dimitrios K. Christodoulou); ${ }^{\mathrm{b}}$ Center for Inflammatory Bowel Diseases, Division of Gastroenterology, BethIsrael Deaconess Medical Center, Harvard Medical School, Boston, Massachusetts (Konstantinos Papamichael); ${ }^{\mathrm{C}}$ Center for Clinical Evidence Synthesis, Tufts Clinical and Translational Science Institute, Tufts University, Boston, MA (Ioannis Koulouridis); ${ }^{\mathrm{d} B e r k s h i r e ~ M e d i c a l ~}$ Center, Pittsfield, MA (Despoina Mavromati)
\end{abstract}

\section{Conflict of Interest: None}

Correspondence to: Dimitrios K. Christodoulou, MD, PhD, Professor of Gastroenterology, Department of Gastroenterology, University Hospital of Ioannina, Faculty of Medicine, School of Health Sciences, University of Ioannina, 45110 Ioannina, Greece, email: dchristodoulou@gmail.com

Received 10 August 2018; accepted 27 December 2018; published online 23 January 2019

DOI: https://doi.org/10.20524/aog.2019.0357

\section{Introduction}

Ulcerative colitis (UC) is a chronic inflammatory bowel disease (IBD) that affects the large intestine and is characterized by alternating periods of flares and remission [1]. To provide patients with the best therapeutic care, a great deal of effort is made to subdivide patients into groups on the basis of recognized risk factors that could act as proxies for aggressive disease, with the goal of early treatment escalation to reduce UC-related complications [2].

There is a subgroup of patients with UC (10-20\%) who present on endoscopy with pseudopolyps, typically formed at the bowel wall during repetitive inflammation attacks $[3,4]$. Pseudopolyps have been associated with an increased burden of disease in the context of intermediate risk of colorectal cancer and the need for increased surveillance [5]. However, as pseudopolyps can be recognized on endoscopy during both periods of remission and flares, and given the fact that not all 
patients with severe inflammation and chronic activity will have pseudopolyps, their role as a marker of IBD activity has been debated $[6,7]$. This, along with the lack of data regarding the long-term outcomes of UC patients with pseudopolyps, is probably why pseudopolyps are not included as part of the scoring system in UC endoscopic scores [8].

This study aimed to investigate treatment escalation, escalation to biological agents or surgery, UC-related hospitalization, and bowel stenosis of patients with UC and pseudopolyps compared with patients without pseudopolyps, and variables associated with these therapeutic outcomes.

\section{Patients and methods}

\section{Study design and population}

This was a single-center retrospective study conducted at the University Hospital of Ioannina, a referral tertiary center for IBD in northwestern Greece. Consecutive patients with UC and available endoscopic data from 2000-2016 were eligible for the study and were followed until June 2017. Data from endoscopies performed to assess disease activity were retrieved for evaluation. Patients with incomplete medical/ endoscopic charts or interrupted follow up were excluded from the study. Patients were divided into two groups: those with pseudopolyps (based on endoscopic and/or histologic criteria) and those without. The study was approved by the hospital's Institutional Ethics Committee.

\section{Outcomes and definitions}

Primary outcomes included treatment escalation, escalation to biological agents or surgery and UC-related hospitalization. Treatment escalation was defined as any difference in treatment between the time of the first colonoscopy (with or without pseudopolyps) and the end of follow up, described as follows: a) from nothing or maintenance therapy with 5-ASA to immunomodulators (IMM) or biological therapy or surgery; b) from IMM to biological therapy or surgery; and c) from biological therapy to colectomy. UC-related hospitalization was defined as any hospitalization due to increased disease activity. Only colectomies performed because of uncontrolled disease were analyzed. A secondary outcome was the association of pseudopolyps with bowel stenosis.

\section{Statistical analysis}

Descriptive statistics were provided with medians and interquartile ranges (IQRs) for continuous variables, and frequencies and percentages for categorical variables. Continuous and discrete variables were compared between groups using the Mann-Whitney $U$ test and the chi-square or Fisher's exact test, as appropriate, respectively. The effect of pseudopolyps on the cumulative probability of treatment escalation, escalation to biological agents or surgery, and UC-related hospitalization was evaluated using time-to-event (survival) methods. KaplanMeier estimates were used to draw the cumulative incidence curves, compared by log-rank test. Univariate and multivariate Cox proportional hazards regression analyses were also performed to determine the independent effects of variables associated with treatment escalation, escalation to biological agents or surgery, and UC-related hospitalization. Univariable and multivariable binary logistic regression analyses were also performed to determine the independent effects of variables associated with bowel stenosis. Variables included sex, age at diagnosis, pancolitis, smoking current or past, corticosteroids at diagnosis, endoscopic Mayo score at diagnosis, and pseudopolyps. Only variables with a P-value $<0.1$ on univariate analysis entered the multivariate analysis, performed using the Wald backward selection method. All analyses were performed using SPSS version 23.0 (SPSS, Chicago, IL, USA) and GraphPad Prism version 5.03 for Windows (GraphPad Software, San Diego, CA, USA).

\section{Results}

\section{Study population}

Of 155 patients initially screened, the study population included 83 patients with UC, of whom 25 (30\%) had pseudopolyps. The median follow up was 2.8 years (IQR 1.1-4.9) and was similar in patients with or without pseudopolyps: 3.4 (IQR 1.3-9.3) vs. 2.6 (IQR 1.1-4.4) years, respectively; $\mathrm{P}=0.341$. The patients' demographic and clinical characteristics are shown in Table 1.

\section{Outcomes}

\section{Treatment escalation}

Overall, 37 (45\%) patients underwent treatment escalation: 17 (68\%) patients with pseudopolyps vs. 20 (34\%) patients without. The probability of treatment escalation was significantly higher in patients with pseudopolyps compared to those without (log-rank $\mathrm{P}=0.011$, Fig. 1A). The first-year cumulative probability for treatment escalation in patients with or without pseudopolyps was $44.5 \%$ (standard error [SE] 0.1) vs. $21.9 \%$ (SE 0.057 ), respectively. The presence of pseudopolyps was the only variable independently associated with treatment escalation (hazard ratio [HR] 2.3, 95\% confidence interval [CI] 1.2-4.3; $\mathrm{P}=0.014$ ) (Table 2A).

\section{Escalation to biological agents or surgery}

Overall, 13/81 (16\%) patients had an escalation to biological agents or surgery at the end of follow up, as two patients were already receiving biologics at baseline: $9 / 23$ (39.1\%) patients 
Table 1 Baseline demographic and clinical characteristics

\begin{tabular}{lcccc}
\hline Characteristics & Total & Patients with pseudopolyps & Patients without pseudopolyps & P-value \\
\hline $\mathrm{N}$ & 83 & 25 & 58 \\
Male sex, (\%) & $46(55)$ & $16(64)$ & $28(48)$ & 0.343 \\
Age at diagnosis, median (IQR), y & $41(27-61)$ & $40(27-62)$ & $43(27-61)$ & 0.846 \\
Pancolitis, (\%) & $37(45)$ & $17(68)$ & $20(34)$ & 0.013 \\
Smoking current or past & $44(53)$ & $15(60)$ & $29(50)$ & 0.476 \\
CS at diagnosis & $31(37)$ & $7(28)$ & 0.325 \\
Endoscopic Mayo score at diagnosis, (\%) & & & $9(0)$ & 0.435 \\
0 & $0(0)$ & $0(0)$ & $36(62)$ & $13(22)$ \\
1 & $12(14)$ & $3(12)$ & \\
2 & $22(59)$ & $9(36)$ & & \\
\hline
\end{tabular}

CS, corticosteroids; IQR, interquartile range; $y$, years
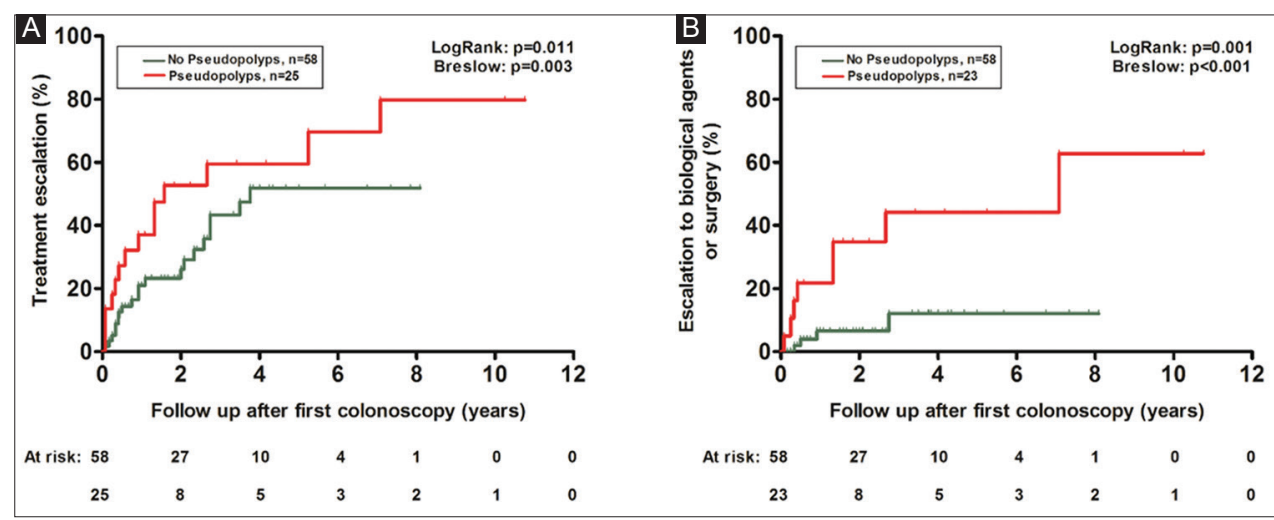

Figure 1 Kaplan-Meier cumulative probability curves for treatment escalation (A) and treatment escalation to biological agents (B) in patients with (red line) or without (gray line) pseudopolyps

with pseudopolyps (biologics, $\mathrm{n}=8$; colectomy, $\mathrm{n}=1$ ) vs. 4/58 (6.9\%) patients without (biologics, $n=3$; colectomy, $n=1$ ). The probability of treatment escalation was significantly higher in patients with pseudopolyps compared to those without (logrank $\mathrm{P}=0.001$, Fig. $1 \mathrm{~B}$ ). The first-year cumulative probability for treatment escalation or surgery in patients with or without pseudopolyps was $25.1 \%$ (SE 0.098) vs. 6.5\% (SE 0.037), respectively. The presence of pseudopolyps was the only variable independently associated with treatment escalation (HR 6.3, 95\%CI 1.9-20.7; $\mathrm{P}=0.002$ ) (Table 2B).

\section{UC-related hospitalization}

During the follow up, 41 (49\%) patients had a UC-related hospitalization: 14 (56\%) patients with pseudopolyps vs. $27(47 \%)$ patients without. The probability of UC-related hospitalization was similar between the two group (log-rank $\mathrm{P}=0.428$, Fig. 2). The first-year cumulative probability for treatment escalation in patients with or without pseudopolyps was $32.5 \%$ (SE 0.095) vs. $27.5 \%$ (SE 0.061), respectively. Pancolitis was the only variable independently associated with UC-related hospitalization (HR 1.9, 95\%CI 1.1-3.6; $\mathrm{P}=0.040$ ) (Table 2C).

\section{Bowel stenosis}

Overall, 8 (9.6\%) patients presented with bowel stenosis on follow-up endoscopy: 5 (20\%) patients with pseudopolyps vs. $3(5.3 \%)$ patients without. Based on univariate analysis the presence of pseudopolyps was marginally associated with bowel stenosis during follow up (odds ratio 4.6, 95\% Cl 1-21; $\mathrm{P}=0.050$, Table 3). A multivariate analysis was not feasible as no other variable was associated with bowel stenosis with a P-value less than 0.1 .

\section{Discussion}

The role of pseudopolyps as a surrogate marker of severe inflammation predicting a more complicated course of IBD is still unclear [9-12]. Intense inflammation and ulceration of mucosa are needed for the formation of pseudopolyps; however, this is considered as a snapshot of severe active disease that lacks the ability to predict the disease course, as there can be a response to induction treatment with sustained remission after initial activity [13]. In this study we demonstrated that the presence of pseudopolyps in patients 
Table 2 Variables associated with treatment escalation (A), escalation to biological agents or surgery (B), and UC-related hospitalization (C)

\begin{tabular}{|c|c|c|c|c|c|c|}
\hline \multirow[t]{2}{*}{ Variables } & \multicolumn{3}{|c|}{ Univariate analysis } & \multicolumn{3}{|c|}{ Multivariate analysis } \\
\hline & $\mathrm{P}$ & HR & $95 \% \mathrm{CI}$ & $\mathrm{P}$ & HR & $95 \% \mathrm{CI}$ \\
\hline \multicolumn{7}{|l|}{ A. Treatment escalation } \\
\hline Pancolitis & 0.059 & 1.9 & $0.9-3.6$ & & & \\
\hline Male sex & 0.240 & 0.7 & $0.3-1.3$ & & & \\
\hline Age at diagnosis & 0.533 & 0.99 & $0.98-1.01$ & & & \\
\hline CS at diagnosis & 0.452 & 1.3 & $0.7-2.5$ & & & \\
\hline Smoking current or past & 0.813 & 1.1 & $0.6-2.1$ & & & \\
\hline Endoscopic Mayo score at diagnosis & 0.666 & 1.1 & $0.7-1.9$ & & & \\
\hline Pseudopolyps & 0.011 & 2.3 & $1.2-4.3$ & 0.014 & 2.3 & $1.2-4.3$ \\
\hline
\end{tabular}

B. Treatment escalation to biological agents or surgery

\begin{tabular}{lccccc}
\hline Pancolitis & 0.054 & 3.2 & $0.98-10.4$ & & \\
Male sex & 0.159 & 0.4 & $0.1-1.4$ & & \\
Age at diagnosis & 0.658 & 0.99 & $0.96-1.02$ & & \\
CS at diagnosis & 0.521 & 1.4 & $0.5-4.3$ & & \\
Smoking current or past & 0.791 & 0.9 & $0.3-2.6$ & & 6.3 \\
\hline Endoscopic Mayo score at diagnosis & 0.941 & 1 & $0.4-2.4$ & & $1.9-20.7$ \\
\hline Pseudopolyps & 0.001 & 6.3 & $1.9-20.7$ & 0.002 & \\
\hline
\end{tabular}

C. UC-related hospitalization

\begin{tabular}{|c|c|c|c|c|c|c|}
\hline Pancolitis & 0.035 & 1.9 & $1.1-3.6$ & 0.040 & 1.9 & $1.1-3.6$ \\
\hline Male sex & 0.788 & 1.1 & $0.6-2$ & & & \\
\hline Age at diagnosis & 0.411 & 1 & $0.99-1.02$ & & & \\
\hline $\mathrm{CS}$ at diagnosis & 0.055 & 1.8 & 0.99-3.4 & & & \\
\hline Smoking current or past & 0.232 & 1,5 & $0.8-2.8$ & & & \\
\hline Endoscopic Mayo score at diagnosis & 0.226 & 1.4 & $0.8-2.3$ & & & \\
\hline Pseudopolyps & 0.434 & 1.3 & $0.7-2.5$ & & & \\
\hline
\end{tabular}

Table 3 Variables associated with bowel stenosis

\begin{tabular}{lccc}
\hline \multirow{2}{*}{ Variables } & \multicolumn{3}{c}{ Univariate analysis } \\
\cline { 2 - 4 } & $\mathrm{P}$ & $\mathrm{OR}$ & $95 \% \mathrm{CI}$ \\
\hline Pancolitis & 0.293 & 2.2 & $0.5-10.1$ \\
Male sex & 0.746 & 1.3 & $0.3-5.5$ \\
\hline Age at diagnosis & 0.302 & 0.99 & $0.98-1.01$ \\
CS at diagnosis & 0.993 & 1 & $0.2-4.5$ \\
\hline Smoking current or past & 0.574 & 1.5 & $0.3-6.9$ \\
Endoscopic Mayo score at diagnosis & 0.541 & 1.4 & $0.4-4.7$ \\
\hline Pseudopolyps & 0.050 & 4.6 & $1-21$ \\
\hline OR, odds ratio; $C$ corticosteroids & & & \\
\hline
\end{tabular}

OR, odds ratio; CS, corticosteroids

with UC was independently associated with a greater need for treatment escalation and escalation to biological agents or surgery, compared to patients with no pseudopolyps. This is of great clinical significance, as patients with pseudopolyps may represent a subgroup of IBD patients with a higher inflammatory burden and more severe disease activity, who need more aggressive treatment including IMM, biologics and surgery.

Reports from recent years suggest that rapid escalation of therapy reduces the IBD burden [14]. This strategy is applied more often to Crohn's disease rather than UC and aims to reduce the structural damage in the bowel wall, creating the concept of a "window of opportunity" for intensive treatment in order to change the natural history of IBD [15]. In UC, the rapid escalation of treatment aims at mucosal healing with a goal of deep remission [16]. Nevertheless, there is a growing body of evidence to suggest that UC may cause structural damage of the bowel wall. An example is the hypertrophy of muscular tissue noticed in cases with longstanding UC [17]. Validated predictors for a more severe course of UC are needed 


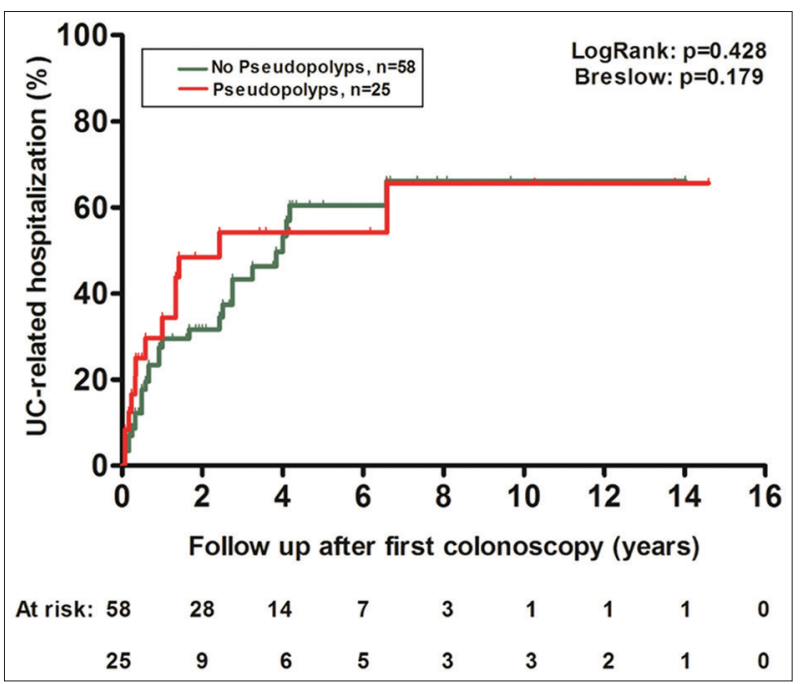

Figure 2 Kaplan-Meier cumulative probability curves for UC-related hospitalization in patients with (red line) or without (gray line) pseudopolyps

UC, ulcerative colitis

to define subgroups of patients who would benefit from intense immunosuppressive treatment, using the same "window of opportunity" concept as in Crohn's disease.

The most widely used endoscopic scores for monitoring UC activity are the Mayo endoscopic subscore, often used in clinical trials, and the Ulcerative Colitis Endoscopic Index of Severity (UCEIS), the first validated endoscopic score of severity in UC. Both scores incorporate similar parameters, such as ulcers, vascular pattern and bleeding, and both have their strengths and weaknesses, proving again that there is not a "perfect" endoscopic tool for assessing UC severity and implying a need for additional endoscopic parameters to improve their performance $[18,19]$.

Pseudopolyps can be found on endoscopy in both periods of flares and remission; they may therefore serve as predictive markers of the natural history of UC in a separate and complementary way to endoscopic parameters describing increasing levels of inflammation. So far, pseudopolyps in endoscopy affect the management of patients with UC only by making them eligible for colorectal cancer monitoring approximately every 3 years [20]. This study implies that the presence of pseudopolyps is linked with a more aggressive disease course requiring rapid treatment escalation, thus suggesting that pseudopolyps may have a place in endoscopic scores. The finding that pseudopolyps may be associated with bowel stenosis advocates for intense endoscopic follow up of patients with pseudopolyps, apart from their known higher risk of colorectal cancer. A hypothetical mechanism to explain this association could include the excessive healing process of the mucosa after ulceration, which may result more often in stenosis and the formation of pseudopolyps.

In our study the presence of pseudopolyps was not associated with a higher risk for UC-related hospitalization, which might appear to conflict with the finding of a higher risk of treatment escalation. This discrepancy may be explained by the rather small sample size and the relatively short follow-up time, which were limitations of the study. Other limitations of the study include its retrospective nature, the potential for selection bias due to the relative high percentage of screening failures, and bias by indication: since our department is a referral center for IBD, more severe and active cases of UC may have been included in our study.

In conclusion, this retrospective single-center study, representing real-life clinical practice, provides the first preliminary evidence that UC patients with pseudopolyps may represent a subpopulation with a higher inflammatory burden and a greater need for treatment escalation, and escalation to biological agents or surgery, compared with patients without pseudopolyps. Large, prospective studies are certainly warranted to confirm these findings.

\section{Summary Box}

\section{What is already known:}

- Pseudopolyps are markers of episodes of severe inflammation, encountered in endoscopy in a subgroup of patients with ulcerative colitis (UC)

- Their clinical significance is uncertain, except for their link with an intermediate risk for colorectal cancer

- Pseudopolyps are not included in endoscopic scores for monitoring the activity of UC

\section{What the new findings are:}

- The presence of pseudopolyps in patients with UC was associated with rapid escalation of treatment, escalation to biological agents or surgery, and possibly with bowel stenosis

- The presence of pseudopolyps in UC patients was not associated with a greater need for hospitalization

- Patients with UC and pseudopolyps may represent a subgroup with a greater burden of disease in terms of increased immunosuppressive treatment

\section{References}

1. Magro F, Gionchetti P, Eliakim R, et al; European Crohn's and Colitis Organisation [ECCO]. Third European Evidence-based Consensus on Diagnosis and Management of Ulcerative Colitis. Part 1: Definitions, diagnosis, extra-intestinal manifestations, pregnancy, cancer surveillance, surgery, and ileo-anal pouch disorders. J Crohns Colitis 2017;11:649-670.

2. Colombel JF, Narula N, Peyrin-Biroulet L. Management strategies to improve outcomes of patients with inflammatory bowel diseases. Gastroenterology 2017;152:351-361.

3. Kelly JK, Gabos S. The pathogenesis of inflammatory polyps. Dis Colon Rectum 1987;30:251-254.

4. Maggs JR, Browning LC, Warren BF, Travis SP. Obstructing giant 
post-inflammatory polyposis in ulcerative colitis: Case report and review of the literature. J Crohns Colitis 2008;2:170-180.

5. Shergill AK, Lightdale JR, Bruining DH, et al; American Society for Gastrointestinal Endoscopy Standards of Practice Committee. The role of endoscopy in inflammatory bowel disease. Gastrointest Endosc 2015;81:1101-1121.e1-e13.

6. Ooi BS, Tjandra JJ, Pedersen JS, Bhathal PS. Giant pseudopolyposis in inflammatory bowel disease. Austr N Z J Surg 2000;70:389-393.

7. De Dombal FT, Watts JM, Watkinson G, Goligher JC. Local complications of ulcerative colitis: stricture, pseudopolyposis, and carcinoma of colon and rectum. Br Med J 1966;1:1442-1447.

8. Annese V, Daperno M, Rutter MD, et al; European Crohn's and Colitis Organisation. European evidence based consensus for endoscopy in inflammatory bowel disease. J Crohns Colitis 2013;7:982-1018.

9. Teague RH, Read AE. Polyposis in ulcerative colitis. Gut 1975;16:792-795.

10. Politis DS, Katsanos KH, Tsianos EV, Christodoulou DK. Pseudopolyps in inflammatory bowel diseases: have we learned enough? World J Gastroenterol 2017;23:1541-1551.

11. Keating JW, Jr., Mindell HJ. Localized giant pseudopolyposis in ulcerative colitis. AJR Am J Roentgenol 1976;126:1178-1180.

12. Choi YS, Suh JP, Lee IT, et al. Regression of giant pseudopolyps in inflammatory bowel disease. J Crohns Colitis 2012;6:240-243.
13. Solberg IC, Lygren I, Jahnsen J, et al; IBSEN Study Group. Clinical course during the first 10 years of ulcerative colitis: results from a population-based inception cohort (IBSEN Study). Scand J Gastroenterol 2009;44:431-440.

14. D'Haens GR. Top-down therapy for IBD: rationale and requisite evidence. Nat Rev Gastroenterol Hepatol 2010;7:86-92.

15. Pariente B, Cosnes J, Danese S, et al. Development of the Crohn's disease digestive damage score, the Lémann score. Inflamm Bowel Dis 2011;17:1415-1422.

16. Lichtenstein GR, Rutgeerts P. Importance of mucosal healing in ulcerative colitis. Inflamm Bowel Dis 2010;16:338-346.

17. Torres J, Billioud V, Sachar DB, Peyrin-Biroulet L, Colombel JF. Ulcerative colitis as a progressive disease: the forgotten evidence. Inflamm Bowel Dis 2012;18:1356-1363.

18. Walsh AJ, Bryant RV, Travis SP. Current best practice for disease activity assessment in IBD. Nat Rev Gastroenterol Hepatol 2016;13:567-579.

19. Mohammed Vashist N, Samaan M, Mosli MH, et al. Endoscopic scoring indices for evaluation of disease activity in ulcerative colitis. Cochrane Database Syst Rev 2018;1:CD011450.

20. Cairns SR, Scholefield JH, Steele RJ, et al; Association of Coloproctology for Great Britain and Ireland. Guidelines for colorectal cancer screening and surveillance in moderate and high risk groups (update from 2002). Gut 2010;59:666-689. 